\title{
Maternal low glycemic index dietary intervention and DNA methylation in 5 year old offspring: results from the ROLO Kids Study
}

\author{
A.A. Geraghty ${ }^{1}$, A. Sexton-Oates ${ }^{2}$, E.C. O'Brien ${ }^{1}$, R. Saffery ${ }^{2,3}$ and F.M. McAuliffe ${ }^{1}$ \\ ${ }^{1}$ UCD Perinatal Research Centre, Obstetrics and Gynaecology, School of Medicine, University College Dublin, \\ National Maternity Hospital, Dublin 2, Ireland, ${ }^{2}$ Cancer and Disease Epigenetics, Murdoch Childrens Research \\ Institute, Melbourne, Victoria, Australia and ${ }^{3}$ Department of Paediatrics, University of Melbourne, Victoria, Australia
}

Environmental factors during fetal development can influence variable DNA methylation patterns which have been implicated in the long-term health of the offspring ${ }^{(1)}$. Maternal diet and nutrition status during pregnancy has been shown to influence offspring DNA methylation at birth but research is limited as to whether these changes persist as the child ages ${ }^{(2)}$. This study aims to investigate if a dietary invention or other maternal factors during pregnancy have a lasting impact on offspring methylation profile at 5 years of age.

Sixty-three 5-year-old children were selected from the ROLO study (Randomised cOntrol trial of LOw glycaemic index diet versus no dietary intervention ${ }^{(3)}$ ). During their pregnancy the mothers were randomised to a low glycemic index dietary intervention or control group and the offspring were followed-up when they turned 5 years of age. Maternal written consent was obtained at both time points. DNA methylation was investigated in 780,501 CpG sites from salivary DNA using the Illumina MethylationEPIC BeadChip array. Principal components analysis (PCA) and linear regression were used to compare the dietary intervention group to controls. Gene pathway analysis was carried out and associations with maternal factors during pregnancy were explored.

PCA identified no relationship between maternal age, weight or BMI during pregnancy with methylation status at 5 years. Regression analyses identified 22,181 differentially methylated CpGs (unadjusted $P \leq 0 \cdot 01$ ) in children of mothers exposed to the dietary intervention compared to the controls. No CpGs remained significant at False-Discovery-Rate corrected p-value. PCA found no strong association with the intervention, nor with birth weight or gestational age. Interestingly, gene pathway analysis identified functional clusters that differed between the intervention and control children, involved in insulin secretion and resistance, pathways targeted by the intervention.

In this modest-sized discovery sample, we identified limited evidence of long-lasting influences of a low glycaemic index dietary intervention in pregnancy on childhood DNA methylation patterns. While pathways related to insulin functioning differed between the groups this was not a primary cause of variation in the sample set. Maternal factors in pregnancy also did not influence the methylome in early childhood. Further research is required to elucidate what impacts long-term methylation status in childhood.

1. Lee (2015) Nutrients 7, 9492-9507.

2. Geraghty, Lindsay, Alberdi et al. (2015) Nutrition and Metabolic Insights 8, 41.

3. Walsh, McGowan, Mahony, et al. (2012) British Medical Journal, 345, e5605-e5605. 